\title{
Singular perturbation methods for slow-fast dynamics
}

\author{
Ferdinand Verhulst
}

Received: 2 February 2006 / Accepted: 4 April 2006 / Published online: 21 February 2007

(C) Springer Science + Business Media B.V. 2007

\begin{abstract}
Recently, geometric singular perturbation theory has been extended considerably while at the same time producing many new applications. We will review a number of aspects relevant to non-linear dynamics to apply this to periodic solutions within slow manifolds and to review a number of non-hyperbolic cases. The results are illustrated by examples.
\end{abstract}

Keywords Singular perturbations $\cdot$ Slow manifolds Periodic solutions $\cdot$ Nonhyperbolic

This paper deals with slow-fast initial value problems that are of the form

$\dot{x}=f(x, y)+\varepsilon \cdots, \quad \varepsilon \dot{y}=g(x, y)+\varepsilon \cdots$,

or alternatively

$\dot{x}=\varepsilon f(x, y)+\varepsilon^{2} \cdots, \quad \dot{y}=g(x, y)+\varepsilon \cdots$.

As usual, $\varepsilon$ is a small positive parameter, and an overdot denotes differentiation with respect to time. For a number of results, the vector fields $f$ and $g$ explicitly depending on time $t$ present no obstruction.

Part of the paper is a tutorial, but there are some new results.

F. Verhulst

Mathematisch Instituut, University of Utrecht, PO Box

80.010, 3508 TA Utrecht, The Netherlands

e-mail: verhulst@math.uu.nl

\section{The Tikhonov theorem}

In singular perturbations, certain attraction (or hyperbolicity) properties of the regular (outer) expansion play an essential part in the construction of the formal approximation. Remarkably enough, this hyperbolicity does not include the behaviour of the slow equation.

In the constructions, the following theorem provides a basic boundary layer property of the solution.

Theorem 1.1. (Tikhonov, 1952, see [15]) Consider the initial value problem

$$
\begin{aligned}
\dot{x}= & f(x, y, t)+\varepsilon \cdots, \quad x(0)=x_{0}, \quad x \in D \subset \mathbb{R}^{n}, \\
& t \geq 0, \\
\varepsilon \dot{y}= & g(x, y, t)+\varepsilon \cdots, \quad y(0)=y_{0}, \quad y \in G \subset \mathbb{R}^{m} .
\end{aligned}
$$

For $f$ and $g$, we take sufficiently smooth vector functions in $x, y$ and $t$; the dots represent (smooth) higherorder terms in $\varepsilon$.

a. We assume that a unique solution of the initial value problem exists and suppose this holds also for the reduced problem

$\dot{x}=f(x, y, t), \quad x(0)=x_{0}$,

$0=g(x, y, t)$,

with solutions $\bar{x}(t), \bar{y}(t)$. 
b. Suppose that $0=g(x, y, t)$ is solved by $\bar{y}=\phi(x, t)$, where $\phi(x, t)$ is a continuous function and an isolated root. Also, suppose that $\bar{y}=\phi(x, t)$ is an asymptotically stable solution of the equation

$$
\frac{d y}{d \tau}=g(x, y, t)
$$

that is uniform in the parameters $x \in D$ and $t \in \mathbb{R}^{+}$.

c. $y(0)$ is contained in an interior subset of the domain of attraction of $\bar{y}=\phi(x, t)$ in the case of the parameter values $x=x(0), t=0$.

Then, we have

$$
\begin{array}{ll}
\lim _{\varepsilon \rightarrow 0} x_{\varepsilon}(t)=\bar{x}(t), & 0 \leq t \leq L, \\
\lim _{\varepsilon \rightarrow 0} y_{\varepsilon}(t)=\bar{y}(t), & 0<d \leq t \leq L
\end{array}
$$

with $d$ and $L$ constants independent of $\varepsilon$.

An interior subset of a domain is a subset of which all the points have a positive distance to the boundary of the domain, which is independent of $\varepsilon$. This condition is necessary to avoid ejection of orbits by an $\varepsilon$-perturbation near the boundary of the domain of attraction.

In assumption (b), $t$ and $x$ are parameters and not variables. The idea is that during the fast motion of the variable $y$, the small variations of these parameters are negligible as long as the stability holds for values of the parameters $x \in D$ and $t \in \mathbb{R}^{+}$.

The uniformity condition in $\mathrm{b}$ and condition $\mathrm{c}$ were not present in the original formulation of the theorem.

Example 1.1. (Restriction of the time-interval) Consider the initial value problem

$$
\begin{aligned}
\ddot{x}+x & =f(x, \dot{x}, y), \quad x(0)=x_{0}, \quad \dot{x}(0)=x_{1}, \\
\varepsilon \dot{y} & =(2 t-1) y g(x), \quad y(0)=y_{0},
\end{aligned}
$$

with $(x, \dot{x}, y) \in \mathbb{R}^{3}, t \geq 0 ; f$ and $g$ are sufficiently smooth, and $g(x)>0$. On the interval $0 \leq t \leq d$ with $0<d<\frac{1}{2}$ (with $d$ a constant independent of $\varepsilon$ ), $y=0$ is an asymptotically stable solution of the equation

$$
\frac{d y}{d \tau}=(2 t-1) y g(x)
$$

with $t, x$ as parameters. We expect a boundary layer in time near $t=0$ after which the solution will settle near the solution of

$\ddot{x}+x=f(x, \dot{x}, 0), \quad y=0, \quad x(0)=x_{0}, \quad \dot{x}(0)=x_{1}$

as long as $0 \leq t \leq d$. Near $t=1 / 2$, another fast transition takes place.

Example 1.2. Consider the two-dimensional autonomous system

$$
\begin{aligned}
\dot{x} & =1, \quad x(0)=x_{0}, \\
\varepsilon \dot{y} & =x y-y^{2}, \quad y(0)=y_{0},
\end{aligned}
$$

with $x, y \in \mathbb{R}, t \geq 0$.

In the case of autonomous (dynamical) systems as in this example, the attracting outer expansions correspond with manifolds in phase-space and so have a clear geometrical meaning. In this case, there are two roots corresponding with "critical points":

$y=0, \quad y=x$.

If $x<0, y=0$ is stable and the second one unstable. If $x>0$, the second one is stable and $y=0$ is unstable.

It follows that for $x<0$ we expect a boundary layer jump towards the $x$-axis $(y=0)$ and for $x>0$ we expect a boundary layer jump towards the line $y=x$. Note, however, the domains of attraction by sketching the phase-plane (see Fig. 1); solutions may also move off to infinity. The manifolds corresponding with the outer expansions, the lines $y=0$ and $y=x$, are called slow manifolds to indicate the difference from the fast behaviour in the boundary layers.

There are two other interesting aspects of this behaviour. In the example, the solutions starting on the left-hand side move on to the right-hand side. On passing the $y$-axis, there is an exchange of stability; there are interesting applications showing such behaviour.

A second interesting aspect is that the slow manifold $y=0$ corresponds with an invariant manifold of the full system; $y=x$, however (excluding a neighbourhood of the origin), is an approximation of a nearby existing invariant manifold. The orbits in the first quadrant of the phase-plane are following the manifold given by $y=x$ closely.

Another interesting point is that to the left of the $y$-axis the solutions get exponentially close to the 


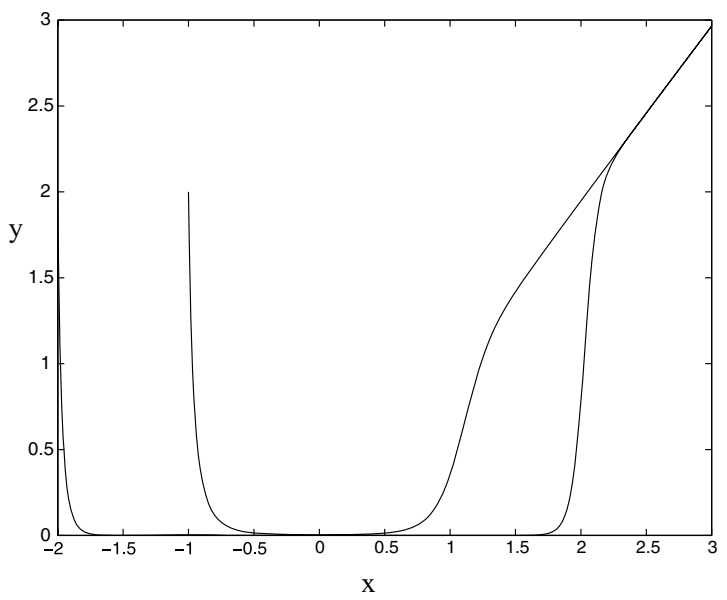

Fig. 1 Solutions in Example 1.2 starting in $(x, y)=(-2,2)$ and $(-1,2), \varepsilon=0.1$. If $x<0$, they are attracted to the slow manifold $y=0$; if $x>0$, the attraction is to the approximate slow manifold $y=x$. Near the $x$-axis, the solutions get exponentially close and the closest one, starting in $(-2,2)$, sticks longer to the $x$-axis after passing a neighbourhood of the origin. If $x(0)<0$, the lift-off is near $-x(0)$

invariant manifold $y=0$. This results in the phenomenon of "sticking to the $x$-axis" for some time after passing the origin. It is not difficult to show that if $x(0)<0$, the lift-off takes place near $-x(0)$.

\section{The O'Malley-Vasil'eva expansion}

How do we use Tikhonov's theorem to obtain approximations of solutions of non-linear initial value problems? The theorem does not state anything about the size of the boundary layer (the parameter $d$ in the theorem) or the timescales involved to describe the initial behaviour and the relative slow behaviour later on.

Asymptotic expansions are described as follows:

Theorem 2.1. (O'Malley-Vasil'eva) Consider the initial value problem in $\mathbb{R}^{n} \times \mathbb{R}^{m} \times \mathbb{R}^{+}$

$$
\begin{aligned}
\dot{x} & =f(x, y, t, \varepsilon), \\
x(0) & =x_{0}, \quad x \in D \subset \mathbb{R}^{n}, \quad t \geq 0, \\
\varepsilon \dot{y} & =g(x, y, t, \varepsilon), \quad y(0)=y_{0}, \quad y \in G \subset \mathbb{R}^{m},
\end{aligned}
$$

where $f$ and $g$ can be expanded in entire powers of $\varepsilon$. Suppose that the requirements of Tikhonov's theorem have been satisfied and moreover that for the solution of the reduced equation $0=g(x, \bar{y}, t, 0), \bar{y}=\phi(x, t)$, we have

$\operatorname{Re}_{\operatorname{Spg}}(x, \bar{y}, t) \leq-\mu<0, \quad x \in D, \quad 0 \leq t \leq L$.

Then, for $t \in[0, L], x \in D, y \in G$, the formal approximation described earlier leads to asymptotic expansions of the form

$$
\begin{aligned}
& x_{\varepsilon}(t)=\sum_{n=0}^{m} \varepsilon^{n} a_{n}(t)+\sum_{n=1}^{m} \varepsilon^{n} \alpha_{n}\left(\frac{t}{\varepsilon}\right)+O\left(\varepsilon^{m+1}\right), \\
& y_{\varepsilon}(t)=\sum_{n=0}^{m} \varepsilon^{n} b_{n}(t)+\sum_{n=0}^{m} \varepsilon^{n} \beta_{n}\left(\frac{t}{\varepsilon}\right)+O\left(\varepsilon^{m+1}\right) .
\end{aligned}
$$

The constant $L$ that bounds the domain of validity in time is in general an $O(1)$ quantity determined by the vector fields $f$ and $g$. For proofs, see [12] and [17]. An intermediate step in the analysis is an expansion of the form

$$
y=\phi(x, t)+\varepsilon y_{1}(x, t)+\varepsilon^{2} y_{2}(x, t)+\varepsilon^{3} \cdots .
$$

The expansion is derived from the fast equation and it is asymptotically valid on a timescale $O(1)$ outside the boundary layer.

\section{The slow manifold: Fenichel's results}

Tikhonov's theorem is concerned with the attraction, at least for some time, to the regular expansion that corresponds with a stable critical point of the (fast) boundary layer equation. The theory is quite general and deals with non-autonomous equations.

In the case of autonomous equations, it is possible to associate with the regular expansion a manifold in phase-space and to consider the attraction properties of the flow near this manifold. This raises the question of whether these manifolds really exist or whether they are just a phantom phenomenon. Such questions were addressed and answered in a number of papers by Fenichel [3-6], and other authors; the reader is referred to the survey papers [9] and [10]; see also [20].

Consider the autonomous system

$$
\begin{aligned}
\dot{x} & =f(x, y)+\varepsilon \cdots, \quad x \in D \subset \mathbb{R}^{n}, \\
\varepsilon \dot{y} & =g(x, y)+\varepsilon \cdots, \quad y \in G \subset \mathbb{R}^{m} .
\end{aligned}
$$


In this context, one often transforms $t \rightarrow \tau=t / \varepsilon$ so that

$$
\begin{aligned}
& x^{\prime}=\varepsilon f(x, y)+\varepsilon^{2} \cdots, \quad x \in D \subset \mathbb{R}^{n}, \\
& y^{\prime}=g(x, y)+\varepsilon \cdots, \quad y \in G \subset \mathbb{R}^{m},
\end{aligned}
$$

where the prime denotes differentiation with respect to $\tau$.

As before, $y$ is called the fast variable and $x$ the slow variable. The zero set of $g(x, y)$ is given again by $y=$ $\phi(x)$, which in this autonomous case represents a firstorder approximation $M_{0}$ of the $n$-dimensional (slow) manifold $M_{\varepsilon}$. The flow on $M_{\varepsilon}$ is to a first approximation described by $\dot{x}=f(x, \phi(x))$.

In Tikhonov's theorem, we assumed asymptotic stability of the approximate slow manifold; in the asymptotic constructions, we assume that the eigenvalues of the linearised flow near $M_{0}$, derived from the equation for $y$, have negative real parts only.

In geometric singular perturbation theory, for which Fenichel's results are basic, we only assume that all real parts of the eigenvalues are non-zero. In this case, the slow manifold $M_{\varepsilon}$ is called normally hyperbolic. A manifold is called hyperbolic if the local linearisation is structurally stable (real parts of eigenvalues all nonzero), and it is normally hyperbolic if in addition the expansion or contraction near the manifold in the transverse direction is larger than in the tangential direction (the slow drift along the slow manifold).

Note that, although this generalisation is not consistent with the asymptotic constructions where all the real parts of the eigenvalues have to be negative, it allows for interesting phenomena. One might approach $M_{\varepsilon}$, for instance, by a stable branch, stay for some time near $M_{\varepsilon}$, and then leave again a neighbourhood of the slow manifold by an unstable branch. This produces solutions indicated as "pulse-like", "multibump solutions", etc. This type of exchanges of the flow near $M_{\varepsilon}$ is what one often looks for in geometric singular perturbation theory.

\section{Note on the size of the time-interval}

If the equations are autonomous, there is no a priori restriction on the interval bound $L$. The restriction of the time interval arises from the conditions that $x$ and $y$ are in the compacta $D$ and $G$. If $x(t)$ leaves $D$ as in Example 1.2, this imposes the bound on the time interval of validity of the estimates. In this example, we also have the phenomenon of the solutions "sticking" to the $x$-axis, even after passing the origin. The explanation is in the exponential closeness to the slow manifold for $x<0$.

\section{Approximations in the slow manifold}

The existence and smoothness of the slow manifold, in combination with the possibility of a regular expansion describing the slow manifold drift, enables us to take a fairly easy shortcut to obtain approximations and, in particular, periodic solutions. Note that if we restrict ourselves to periodic solutions within a slow manifold, this excludes the case of non-hyperbolic transition as found in relaxation oscillations.

We will develop the following setup of a theorem leading to periodic solutions. Using the wealth of results on periodic solutions by averaging and normalisation, it is not difficult to develop this idea to other cases.

Consider the autonomous system in $\mathbb{R}^{n} \times \mathbb{R}^{m} \times \mathbb{R}^{+}$

$$
\begin{aligned}
\dot{x}= & f_{0}(x, y)+\varepsilon f_{1}(x, y)+\varepsilon^{2} \cdots, \quad x \in D \subset \mathbb{R}^{n}, \\
& t \geq 0, \\
\varepsilon \dot{y}= & g_{0}(x, y)+\varepsilon g_{1}(x, y)+\varepsilon^{2} \cdots, \quad y \in G \subset \mathbb{R}^{m},
\end{aligned}
$$

where $f_{0}, f_{1}, g_{0}, g_{1}$ are smooth vector functions, the dots represent higher order terms. Furthermore the assumptions of Tikhonov's and Fenichel's theorems apply for $0 \leq t \leq L$.

For the solutions in the slow manifold, we can apply the expansion (1) $y=\phi(x)+\varepsilon y_{1}(x)+\varepsilon^{2} \ldots$ with $g_{0}(x, \phi(x))=0$.

For $x(t)$ in the slow manifold, this results in

$$
\begin{aligned}
\dot{x}= & f_{0}(x, \phi(x))+\varepsilon \frac{\partial f_{0}}{\partial y}(x, \phi(x)) y_{1}(x) \\
& +\varepsilon f_{1}(x, \phi(x))+\varepsilon^{2} \cdots .
\end{aligned}
$$

This is still a very general system and much depends on the explicit solvability of the reduced equation which arises for $\varepsilon=0$. Note that if we would strictly apply the O'Malley-Vasil'eva expansion for the equations governing the slow manifold flow, this may produce secular terms when approximating periodic solutions.

A relatively simple result arises as follows. Suppose that putting $\varepsilon=0$, the slow manifold Equation (2) 
reduces to

$\dot{z}=A z$,

with $A$ a constant $n \times n$-matrix with purely imaginary eigenvalues only. The solution of the reduced equation is given by

$z(t)=\Phi(t)$.

$\Phi(t)$ is a fundamental matrix which is quasiperiodic or $T$-periodic.

In the $T$-periodic case (condition $T<L$ ), we can apply to Equation (2) the Poincaré-Lindstedt method. Moreover, in general, we can apply averaging. If the averaged vector field in the $T$-periodic case has a critical point with only one eigenvalue with real part zero, we can apply the Bogoliubov-Mitropolsky theorem to obtain the existence and approximation of a periodic solution; for examples and proofs of the Poincaré-Lindstedt and the Bogoliubov-Mitropolsky theorems, see [18].

Example 4.1. Consider the system

$$
\begin{aligned}
\ddot{x}+x & =\varepsilon\left(2-x^{2}-y^{2}\right) \dot{x}+\varepsilon^{2} \cdots, \\
\varepsilon \dot{y} & =x-y+\varepsilon \cdots,
\end{aligned}
$$

in which the dots represent smooth higher order perturbations. Using the expansion $y=x+\varepsilon \cdots$, we find that the behaviour of $x(t)$ in the slow manifold is governed by the equation

$\ddot{x}+x=2 \varepsilon\left(1-x^{2}\right) \dot{x}+\varepsilon^{2} \ldots$.

To $O(\varepsilon)$, this equation contains a hyperbolic periodic solution (from the van der Pol-equation), which, therefore, persists under higher order autonomous perturbations.

Note that classical theorems as in [1] and [7] do not produce such a result on the existence and approximation of a periodic solution because the reduced equation $(\varepsilon=0)$ is not structurally stable.

\section{Non-hyperbolic transitions}

Transitions arising from non-hyperbolicity have been studied in various contexts. We discuss a few important cases.

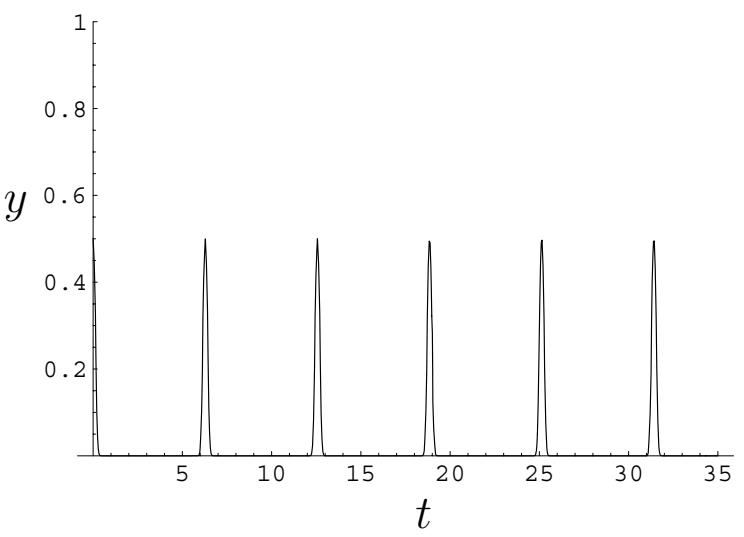

Fig. 2 The solution $y(t)$ of the equation $\varepsilon \dot{y}=-x y(1-$ $y), y(0)=0.5$ with $x(t)=\sin t$ which starts being attracted to the slow manifold $y=0$. Although the stability between the slow manifolds $y=0$ and $y=1$ changes periodically, exponential sticking delays the departure from $y=0$ and produces spike behaviour; $\varepsilon=0.01$

\subsection{Jumping phenomena}

Interesting phenomena take place when the solutions jump repeatedly from one manifold to another, nonintersecting one. This may give rise to periodic solutions or oscillations with fast and slow motions. A simple example runs as follows.

\section{Example 5.1. Consider the system}

$$
\begin{aligned}
\dot{x} & =f(x, y), \\
\varepsilon \dot{y} & =-x y(1-y) .
\end{aligned}
$$

We assume that the equation for $x$ contains oscillatory solutions. As an illustration, we replace it by an explicit function, $\sin t$. The slow manifolds are $y=0$ and $y=1$ with eigenvalues, respectively, $-x$ and $x$.

Choosing periodic behaviour of $x(t)$ we find spikelike relaxation behaviour as shown in Fig. 2. This is quite surprising as one would expect that the stability transitions would produce blocklike oscillations between $y=0$ and $y=1$. The reason for this spike behaviour is that when the slow manifold becomes unstable, the solutions are exponentially close to the unstable slow manifold. It takes an $O(1)$ time-interval to leave this manifold and around that time, there is again a transition to stability.

Similar phenomena can be found when $x(t)$ is chosen as a quasi-periodic or almost-periodic function. 


\subsection{Relaxation oscillations and quenching}

Relaxation oscillations are classical phenomena where jumps, fast transitions, take place after motion along a slow manifold that has become unstable. For this topic, see [8, 11] and [14]. Most rigorous analysis is carried out for two-dimensional autonomous and forced problems and it is not easy to extend this to more dimensions. As an example, we will discuss briefly a four-dimensional problem from [19] where the evidence is partly numerical. The classical example is the van der Pol relaxation equation.

Quenching of undesirable relaxation oscillations by energy absorption or exchange of energy can take different forms. Apart from straightforward damping mechanisms, one can use autoparametric coupling which we shall discuss here. A general characterisation of autoparametric systems is given in [16], see also [2] and [13].

The important questions are 'what are the conditions for the frequencies of the coupled oscillators' and 'what are the requirements for the coupling terms to achieve effective destabilisation of the normal mode' (or, more modestly, 'reduction of the amplitude of the normal mode').

It turns out that to destabilise relaxation oscillations one needs, apart from correct tuning, rather strong interactions of a special form. This is tied in with the necessity to perturb the slow manifold which characterises to a large extent the relaxation oscillation. The results of this example are discussed in more detail in [19].

Example 5.2. Consider the van der Pol equation

$\ddot{x}+x=\mu\left(1-x^{2}\right) \dot{x}, \quad \mu \gg 0$.

Pure van der Pol relaxation is illustrated in Fig. 3 using Liénard variables.

Consider the possibility of quenching the van der Pol relaxation oscillator by embedding it in an autoparametric system of the form

$$
\begin{aligned}
\ddot{x}+x & =\mu\left(1-x^{2}\right) \dot{x}+\mu c \dot{x} y^{2}, \\
\ddot{y}+\kappa \dot{y}+q^{2} y & =d x y .
\end{aligned}
$$

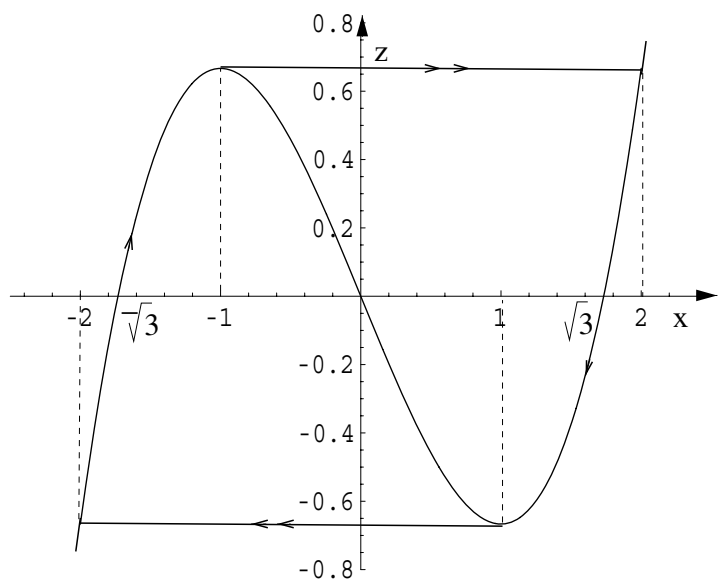

Fig. 3 The phase plane of pure van der Pol relaxation in Liénard variables. The slow manifold is approximated by a cubic curve, fast motion is indicated by double arrows. The slow manifold is unstable for $-1<x<+1$

In generalised Liénard variables, this becomes

$$
\begin{aligned}
\frac{1}{\mu} \dot{x} & =z+x-\frac{1}{3} x^{3}+c x y^{2}, \\
\dot{z} & =-\frac{1}{\mu} x-2 c x y \dot{y}
\end{aligned}
$$

with the equation for $y$ added. The slow manifold is given by

$z=-\left(1+c y^{2}\right) x+\frac{1}{3} x^{3}$,

which is unstable if $1+c y^{2}-x^{2}>0$. The slow manifold corresponds with a 3 -dimensional cubic cylinder parallel to the $\dot{y}$-axis.

This is a rich system with coexisting periodic and chaotic behaviour. As a numerical illustration for the behaviour of the dynamics of system (3-4), we present a projection of the limit set on the $(x, \dot{x})$-plane (so the transients were left out) in Fig. 4. For more numerical experiments, see [19].

\subsection{A remark on resonance manifolds}

In mechanics, an important part is played by slow-fast sytems, usually called amplitude-angle or action-angle systems, of the form

$\dot{x}=\varepsilon X(\phi, x)+\varepsilon^{2} \cdots, \dot{\phi}=\Omega(x)+\varepsilon \cdots$, 


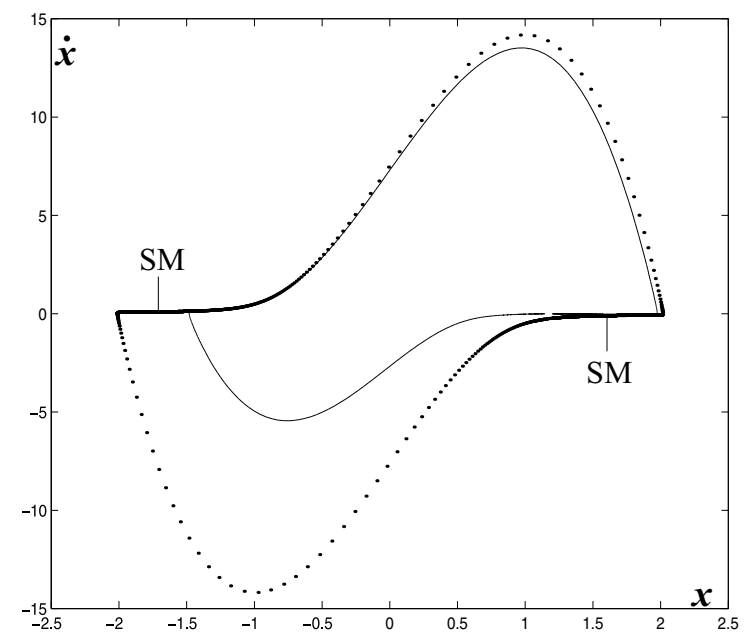

Fig. 4 A periodic limit set of the system (3-4) for $\mu=10, c=$ $-2.2, d=0.03, \kappa=0.075$ with high starting values of the $y$ oscillation, projected on the $x-\dot{x}$ plane. The dotted orbit corresponds with the unperturbed van der Pol relaxation oscillation. In the perturbed state, the slow manifolds are reduced and the limit cycle becomes asymmetric. SM is the stable part of the slow manifold

with $x \in \mathbb{R}^{n}, \phi \in \mathbb{T}^{m}$. The so-called spatial variable $x$ is derived from a system of oscillators, $\phi$ indicates the corresponding angles, defined on the $m$-torus. Averaging over the torus (the angles) is possible outside the resonance manifolds. The latter correspond with the zeros of the right-hand side of the equation for $\phi$ written out in all the possible combination angles.

However, it is already clear that, because of the form of the right-hand side of the angle equation, such a resonance manifold will not be hyperbolic. One might expect, that localising around such a resonance manifold might resolve this, but it is shown quite generally in [18], Section 11.7 that to first order, the equations determining the dynamics in the resonance manifold are not structurally stable, even if the original oscillator system is dissipative. As second-order approximation produces hyperbolicity in a number of cases, we conclude that slow-fast systems of this type are of a different nature and have only a superficial similarity to systems with slow manifolds of Fenichel type.

\section{References}

1. Anosov, D.V.: On limit cycles in systems of differential equations with a small parameter in the highest derivatives. AMS Transl. Ser. 2(33), 233-276 (1963)

2. Cartmell, M.: Introduction to Linear, Parametric and Nonlinear Vibrations. Chapman \& Hall, London (1990)

3. Fenichel, N.: Persistence and smoothness of invariant manifolds for flows. Ind. Univ. Math. J. 21, 193-225 (1971)

4. Fenichel, N.: Asymptotic stability with rate conditions. Ind. Univ. Math. J. 23, 1109-1137 (1974)

5. Fenichel, N.: Asymptotic stability with rate conditions, II. Ind. Univ. Math. J. 26, 81-93 (1977)

6. Fenichel, N.: Geometric singular perturbations theory for ordinary differential equations. J. Differ. Equ. 31, 53-98 (1979)

7. Flatto, L., Levinson, N.: Periodic solutions of singularly perturbed systems. J. Ration. Mech. Anal. 4, 943-950 (1955)

8. Grasman, J.: Asymptotic Methods for Relaxation Oscillations and Applications, p. 222, Springer-Verlag, New York (1987)

9. Jones, C.K.R.T.: Geometric singular perturbation theory. In: Dynamical Systems, Montecatini Terme, Lecture Notes in Mathematics, Johnson, R. (ed.), Springer-Verlag, Berlin, vol. 1609, pp. 44-118 (1994)

10. Kaper, T.J.: An introduction to geometric methods and dynamical systems theory for singular perturbation problems. In: Analyzing Multiscale Phenomena Using Singular Perturbation Methods, Proceedings of the Symposium on Applied Mathematics, Cronin, J., O’Malley Jr., R. E. (eds.), American Mathematical Society, Providence, RI, vol. 56, pp. 85-131 (1999)

11. Krupa, M., Szmolyan, P.: Relaxation oscillation and canard explosion. J. Differ. Equ. 174, 312-368 (2001)

12. O'Malley, R.E. Jr.: Introduction to Singular Perturbations. Academic, New York (1974)

13. Schmidt G., Tondl, A.: Non-linear Vibrations, p. 420, Akademie-Verlag, Berlin (1986)

14. Szmolyan, P., Wechselberger, M.: Relaxation oscillations in $\mathbb{R}^{3}$. J. Differ. Equ. 200, 69-104 (2004)

15. Tikhonov, A.N.: Systems of differential equations containing a small parameter multiplying the derivative. Mat. Sb. 31, 575-586 (1952)

16. Tondl, A., Ruijgrok, T., Verhulst, F., Nabergoj, R.: Autoparametric Resonance in Mechanical Systems, p. 196, Cambridge University Press, New York (2000)

17. Vasil'eva, A.B.: Asymptotic behaviour of solutions to certain problems involving nonlinear differential equations containing a small parameter multiplying the highest derivatives. Russ. Math. Surv. 18, 13-84 (1963)

18. Verhulst, F.: Nonlinear Differential Equations and Dynamical Systems, p. 304, Springer-Verlag, New York (2000)

19. Verhulst, F., Abadi: Autoparametric resonance of relaxation oscilations. ZAMM 85, 122-131 (2005)

20. Verhulst, F.: Methods and Applications of Singular Perturbations, Boundary Layers and Multiple Timescale Dynamics, p. 340, Springer-Verlag, Berlin (2005) 